\title{
DOMINATING SETS FOR CONVEX FUNCTIONS WITH SOME APPLICATIONS
}

\author{
E. CARRIZOSA
}

J.B.G. FRENK

October 8, 1996

\begin{abstract}
A number of optimization methods require as a first step the construction of a dominating set (a set containing an optimal solution) enjoying properties such as compactness or convexity.

In this note we address the problem of constructing dominating sets for problems whose objective is a componentwise nondecreasing function of (possibly an infinite number of) convex functions, and we show how to obtain a convex dominating set in terms of dominating sets of simpler problems.

The applicability of the results obtained is illustrated with the statement of new localization results in the fields of Linear Regression and Location.
\end{abstract}

Keywords: Dominating set, Convexity, Regression, Location.

\section{Introduction}

Let $I$ be an arbitrary index set and denote by $\prod_{i \in I} A_{i}$ the Cartesian product of the nonempty sets $A_{i}, i \in I$. If $X \subset \mathbb{R}^{n}$ is a nonempty closed convex set and $\Phi: \prod_{i \in I} \mathbb{R} \longrightarrow \mathbb{R}$ is a componentwise nondecreasing function, consider then the optimization problem

$$
\begin{array}{ll}
\inf & \Phi\left(\left(f_{i}(x)\right)_{i \in I}\right), \\
\text { s.t. } & x \in X
\end{array}
$$

where $\left\{f_{i}\right\}_{i \in I}$ is a collection of finite valued functions on $\mathbb{R}^{n}$.

For simplicity it is also assumed that the infimum $v\left(P_{\Phi}\right)$ is finite, although not necessarily attained. 
Such optimization problems occur for example in the fields of Location and Linear Regression.

In order to solve these problems a number of existing algorithms require, as a preliminary step, the construction of a compact convex set or even a polytope which contains an optimal solution to $\left(P_{\Phi}\right)$. To mention a few, consider the cutting plane (e.g. [1]) or ellipsoid methods (e.g. [2, 3, 4]) of (quasi)convex analysis, as well as many strategies of Global Optimization, such as Branch and Bound procedures (e.g. [5]).

Although the feasible set $X$ may serve as a dominating set in some cases, this is not the general rule. In unconstrained problems, for instance, a compact dominating set is required, and this may rule out the set $X$.

In the absence of a good knowledge of the behavior of the function $\Phi$ (apart from its nondecreasing character), a plausible strategy would consist of finding dominating sets which are just determined by the functions $f_{i}$, thus independent of $\Phi$ (see e.g. [6]).

The aim of this paper is to show how, under suitable assumptions, such dominating sets can be constructed from dominating sets for simpler problems (involving a much lower number of functions $f_{i}$ ). This approach seems to be especially useful to cope with problems where the cardinality of $I$ is much higher than the dimension of the space, as, for instance, for many problems occurring in Location Theory or Statistical Estimation.

Throughout the paper $|A|$ denotes the cardinality of the set $A, \operatorname{conv}(B)$ the convex hull of $B$, and $\operatorname{clconv}(B)$ denotes the closure of conv $(B)$. Furthermore, it is assumed that $|I| \geq n$.

\section{Dominance}

To start our analysis of dominating sets we first introduce its definition, $[6,7,8]$.

Definition 2.1 If $J \subset I$, the point $y \in X J$-dominates the point $x \in X$ if $f_{j}(y) \leq f_{j}(x)$ for all $j \in J$. Moreover, the set $K \subset X$ is called a $J-$ dominating set if for every $x \in X$ there exists some $y \in K J$-dominating $x$.

Introducing for every nonempty $J \subset I$ and every $x \in X$ the nonempty set $D_{J}(x)$ given by

$$
D_{J}(x):=\left\{y \in X: f_{j}(y) \leq f_{j}(x) \forall j \in J\right\},
$$

it follows by the above definition that $K \subset X$ is a $J$-dominating set if and only if $K \cap D_{J}(x)$ is nonempty for every $x \in X$.

Let now $\mathcal{K}_{J}, J \subseteq I$, denote the set of $J$-dominating sets. Since $X$ is a $J$-dominating set we obtain that $\mathcal{K}_{J}$ is always nonempty. 
Before showing the important role of $I$-dominating sets we need the following definition.

Definition 2.2 Given $\varepsilon \geq 0$, the point $x \in X$ is called an $\varepsilon$-optimal solution of the optimization problem $\left(P_{\Phi}\right)$ if $v\left(P_{\Phi}\right) \geq \Phi\left(\left(f_{i}(x)\right)_{i \in I}\right)-\varepsilon$

Observe, for $\varepsilon=0$, one obtains the standard definition of optimal solution. On the other hand, by the definition of an infimum, it is clear that the set of $\varepsilon$-optimal solutions is always nonempty for every $\varepsilon>0$.

The following result relates $I$-dominating sets and $\varepsilon$-optimal solutions.

LEMMA 2.1 If $\Phi$ is nondecreasing, then each $K \in \mathcal{K}_{I}$ contains an E-optimal solution of $\left(P_{\Phi}\right)$ for every $\varepsilon>0$. Moreover, if the set of optimal solutions is nonempty, then $K$ contains an optimal solution.

Proof. If $x^{*}$ is an $\varepsilon$-optimal solution for a given $\varepsilon \geq 0$, then the nondecreasing character of $\Phi$ implies that any element in $D_{I}\left(x^{*}\right)$ is also $\varepsilon$-optimal. In particular, any $x \in K \cap D_{I}\left(x^{*}\right)$ is $\varepsilon$-optimal.

To continue our analysis we introduce the following definition.

DEFINITION 2.3 The function $f: X \rightarrow \mathbb{R}$ is called inf-bounded if for every $r \in \mathbb{R}$ the lower level set $\{x \in X: f(x) \leq r\}$ is bounded.

As observed in the introduction we are interested in constructing an element of $K_{I}$ which is compact, convex and contains an optimal solution of $\left(P_{\Phi}\right)$. To find such an element we first need to know whether the set

$$
\hat{K}_{I}:=\left\{K \in K_{I}: K \text { compact and convex }\right\}
$$

is nonempty. For $I$ a finite set and $f_{i}, i \in I$, inf-bounded it is easy to verify that the set clconv $\left(\left(\bigcup_{i \in I} D_{\{i\}}(x)\right)\right.$, with $x \in \mathbb{R}^{n}$ arbitrarily chosen, belongs to $\hat{K}_{I}$. However, for $I$ infinite it seems to be difficult to come up with an easy verifiable condition which quarantees that the set $\hat{K}_{I}$ is nonempty. Therefore, for the general case we only show in Theorem 2.2 a procedure which generates a closed convex set belonging to $K_{I}$. Depending upon the specific example $\left(P_{\Phi}\right)$ under consideration this procedure generates a bounded (and hence compact) or unbounded convex set. On the other hand, if we were succesfull generating any $K$ belonging to $\hat{K}_{I}$ it follows for $I$ finite or countably infinite that this set automatically contains an optimal solution of $\left(P_{\Phi}\right)$ under some weak topological properties on the function $\Phi$ and $f_{i}, i \in I$. To prove this, we need to introduce the following well-known definition (see $[9]$ ).

Definition 2.4 Let $M$ be a metric space. A function $h: M \rightarrow \mathbb{R}$ is called lower semicontinuous on $M$ if for every $x \in M$ it follows that $\liminf _{y \rightarrow x} f(y)=f(x)$. 
If $I$ equals the set of natural numbers then the product space $\prod_{i \in I} I R$ equipped with the weak topology generated by the projections $\pi_{i}, i \in I$ is metrizable, and its metric is given by (see page 8 of [10])

$$
\mathrm{d}(x, y)=\sum_{i=1}^{\infty} 2^{-i}\left|\pi_{i}(x)-\pi_{i}(y)\right| .
$$

Hence the set $\prod_{i \in I} \mathbb{R}$ is a metric space with metric $\mathrm{d}(\cdot, \cdot)$ and so the conditions in the next lemma are properly defined.

LEMMA 2.2 If the set $I$ is finite or countably infinite, the functions $f_{i}$ are lower semicontinuous and the componentwise-nondecreasing function $\Phi$ is also lower semicontinuous on $\prod_{i \in I} I R$ then the function $x \mapsto \Phi\left(\left(f_{i}(x)\right)_{i \in I}\right)$ is lower semicontinuous on $\mathbb{R}^{n}$.

Proof. By Proposition 1.4 of [9] one needs to show that the epigraph epi $(h)=\left\{(x, r) \in \mathbb{R}^{n+1}: h(x) \leq r\right\}$, with $h(x)=\Phi\left(\left(f_{i}(x)\right)_{i \in I}\right)$ is a closed set. Consider therefore a sequence $\left\{\left(x_{k}, r_{k}\right)\right\}_{k \in N}$ belonging to epi $(h)$ and suppose it converges to $(x, r)$. Since $\Phi$ is componentwise nondecreasing this implies with $\varepsilon_{k}:=\left\|x-x_{k}\right\|$ and $\|\cdot\|$ the Euclidean norm that

$$
\begin{aligned}
r & =\liminf _{k \rightarrow \infty} r_{k} \\
& \geq \liminf _{k \rightarrow \infty} \Phi\left(\left(f_{i}\left(x_{k}\right)\right)_{i \in I}\right) \\
& \geq \liminf _{k \rightarrow \infty} \Phi\left(\left(\inf \left\{f_{i}(y): y \in x+\varepsilon_{k} B\right\}\right)_{i \in I}\right),
\end{aligned}
$$

where $B$ denotes the closed Euclidean unit ball. Applying now the lower semicontinuity of the functions $f_{i}$ it follows for every $i \in I$ that

$$
\lim _{k \rightarrow \infty} \inf \left\{f_{i}(y): y \in x+\varepsilon_{k} B\right\}=f_{i}(x),
$$

and so the vector $\left(\inf \left\{f_{i}(y): y \in x+\varepsilon_{k} B\right\}\right)_{i \in I}$ converges in the metric $d$ of $\prod_{i \in I} \mathbb{R}$ to $\left(f_{i}(x)\right)_{i \in I}$. Finally by the lower semicontinuity of the function $\Phi$ and (1) we obtain that

$$
\begin{aligned}
r & \geq \lim \inf _{k \rightarrow \infty} \Phi\left(\left(\inf \left\{f_{i}(y): y \in x+\varepsilon_{k} B\right\}\right)_{i \in I}\right) \\
& \geq \Phi\left(\left(f_{i}(x)\right)_{i \in I}\right),
\end{aligned}
$$

and this proves the desired result.

By Lemmas 2.1 and 2.2 and Weierstrass theorem the next existence result follows immediately.

THEOREM 2.1 If I is a finite or countably infinite index set and the functions $\Phi$ and $\left(f_{i}\right)_{i \in I}$ are lower semicontinuous then the existence of a compact I-dominating set implies that the set of optimal solutions of $\left(P_{\Phi}\right)$ is nonempty. Moreover, any compact I-dominating set contains an optimal solution of $\left(P_{\Phi}\right)$. 
Proof. By Lemma 2.1 it follows for any compact $I$-dominating set $K$ that $v\left(P_{\Phi}\right)=\inf _{x \in K} \Phi\left(\left(f_{i}(x)\right)_{i \in I}\right)$. Applying now Weierstrass theorem (see corollary 1.2 of [9]) and Lemma 2.2 we obtain that $v\left(P_{\Phi}\right)=\min _{x \in K} \Phi\left(\left(f_{i}(x)\right)_{i \in I}\right)$ and this proves the desired result.

In the remainder we always assume that the functions $\Phi$ and $\left(f_{i}\right)_{i \in I}$ are lower semicontinuous. This implies by Theorem 2.1 for $I$ finite or countably infinite that after the construction of a compact $I$-dominating set it automatically contains an optimal solution and so this set can indeed serve as a starting set of one of the algorithms mentioned in the introduction. Therefore the remainder of this section is devoted to the construction of $I$-dominating sets.

THEOREM 2.2 Suppose for every $i \in I$ that the functions $f_{i}$ are finite valued and convex, and there exists at least one $i \in I$ such that $f_{i}$ is inf-bounded. If for every $J \subset I$ with $|J|=n$ one can find some $K_{J} \in \mathcal{K}_{J}$ then

$$
\operatorname{clconv}\left(\cup_{|J|=n} K_{J}\right) \in \mathcal{K}_{I} .
$$

Moreover, if $\cup_{|J|=n} K_{J}$ is a bounded set then clconv $\left(\cup_{|J|=n} K_{J}\right)$ is compact.

Proof. Let $K:=\operatorname{clconv}\left(\cup_{|J|=n} K_{J}\right)$, and suppose that $K \notin \mathcal{K}_{I}$. If this holds then there exists some $x \in X$ such that

$$
\begin{aligned}
\emptyset & =D_{I}(x) \cap K \\
& =\cap_{i \in I}\left(D_{\{i\}}(x) \cap K\right)
\end{aligned}
$$

By our assumptions it follows that the functions $f_{i}$ are continuous and so the collection $\left\{K,\left(D_{\{i\}}(x)\right)_{i \in I}\right\}$ is a collection of closed convex sets of which at least one is compact. Since $x \in \cap_{i \in I} D_{\{i\}}(x)$ this implies by Helly's theorem (see the remark after Corollary 21.3.2 of [11]) that there exists a subset $J \subset I$ with $|J|=n$, such that

$$
\begin{aligned}
\emptyset & =\cap_{j \in J}\left(D_{\{j\}}(x) \cap K\right) \\
& =D_{J}(x) \cap K .
\end{aligned}
$$

However it follows by the definition of $K$ that

$$
D_{J}(x) \cap K \supset D_{J}(x) \cap K_{J} \neq \emptyset,
$$

and this yields a contradiction. Finally, the last part is a direct consequence of Theorem III.1.4.3 of [12].

For Theorem 2.2 to hold it is sufficient to assume that the lower level sets of the functions $f_{i}$ are closed and convex and so we may impose the weaker assumption that the functions $f_{i}$ are finite-valued, lower semicontinuous and quasiconvex. However, we cannot remove the inf-boundedness assumption as shown by the following counterexample. 
Example 2.1 Let $X=\mathbb{R}^{2}, I=I N$ and for each $i \in I$, let $f_{i}: \mathbb{R}^{2} \longrightarrow \mathbb{R}$ be given by

$$
f_{i}\left(x_{1}, x_{2}\right)=\left\{\begin{array}{lc}
0, & \text { if } \quad 0 \leq x_{1} \leq(1 / i) x_{2} \\
1, & \text { otherwise }
\end{array}\right.
$$

Clearly the functions $f_{i}, i \in \mathbb{N}$ are lower semicontinuous and quasiconvex. If $K=\{(1, t): t \geq 0\}$, then $K$ is a closed convex set and it is easy to verify that $K$ belongs to $\mathcal{K}_{J}$ for every finite set $J$. Therefore, if Theorem 2.2 would be valid then $K$ should also belong to $\mathcal{K}_{I}$ but this is not true due to

$$
D_{I}((0,0))=\{(0, t): t \geq 0\} \text {. }
$$

If the set $I$ is finite, one can prove without the inf-boundedness assumption a similar result as discussed by Theorem 2.2 .

THEOREM 2.3 Let I be finite, and suppose for every $i \in I$ that the functions $f_{i}$ are finite valued and convex. If for each $J \subset I$ with $|J|=n$ one can find some $K_{J} \in \mathcal{K}_{J}$ then

$$
\operatorname{conv}\left(\cup_{|J|=n} K_{J}\right) \in \mathcal{K}_{I}
$$

Moreover, if each $K_{J}$ is compact then $\operatorname{conv}\left(\cup_{|J|=n} K_{J}\right)$ is also compact.

Proof. The first part follows immediately by Theorem 21.6 of [11] and the first part of the proof of Theorem 2.2. Moreover, if the set $K_{J}$ for every set $J$ with $|J|=n$ is compact it follows that $\cup_{|J|=n} K_{J}$ is also compact and by Theorem III.1.4.3 of [12] the second part follows.

By Theorem 2.3 for sets $I$ with $|I|=m$ much larger than $n$ the construction of a compact $I$-dominating set is reduced to the construction of a compact $J$-dominating set for each $J$ with $|J|=n$. Although we need $\left(\begin{array}{c}m \\ n\end{array}\right)$ different $J$-dominating sets, it will turn out that this is relatively easy in some applications. We will now focus on whether a given compact and convex set is actually $J$-dominating with $J \subset I$ and $|J|=n$. To decide this we introduce the optimization problem $P_{\lambda}$ given by

$$
\min _{x \in X} \sum_{j \in J} \lambda_{j} f_{j}(x)
$$

It is now possible to prove the next result.

THEOREM 2.4 Let $J$ be a nonempty finite set and $f_{j}, j \in J$, a collection of finite-valued convex functions. If $K \subset X$ is a compact and convex set such that $K$ contains an optimal solution of $\left(P_{\lambda}\right)$ for every $\lambda=\left(\lambda_{j}\right)_{j \in J}$ with $\lambda_{j}>0, j \in J$, then $K$ is a $J$-dominating set. 
Proof. If $K$ does not belong to $K_{J}$ then there exists some $x^{*} \in X$ such that $K \cap D_{J}\left(x^{*}\right)$ is empty. Introduce now the vector-valued function $F$ : $\mathbb{R}^{n} \rightarrow \mathbb{R}^{|J|}$ given by $F(x)=\left(f_{j}(x)\right)_{j \in J}$. Since $K \cap D_{J}\left(x^{*}\right)$ is empty this implies that $F\left(x^{*}\right)$ does not belong to $F(K)+\mathbb{R}_{+}^{|J|}$. Moreover, by the convexity of $f_{j}, j \in J$, we obtain that $F(K)+\mathbb{R}_{+}^{|J|}$ is a convex set. Also, by the continuity of $F$ and $K$ compact the set $F(K)$ is compact and so it follows that $F(K)+\mathbb{R}_{+}^{|J|}$ is a closed set. Hence, by a well-known separating hyperplane result between a point and a closed set not containing this point, [11], there exists some $\delta>0$ and $\lambda=\left(\lambda_{j}\right)_{j \in J} \neq 0$ satisfying

$$
\sum_{j \in J} \lambda_{j} z_{j} \geq \delta+\sum_{j \in J} \lambda_{j} f_{j}\left(x^{*}\right)
$$

for every $z=\left(z_{j}\right)_{j \in J}$ belonging to $F(K)+\mathbb{R}_{+}^{|J|}$. It is now easy to show that $\lambda_{j} \geq 0$ for every $j \in J$ and by the above inequality we obtain that

$$
\sum_{j \in J} \lambda_{j} f_{j}(y) \geq \delta+\sum_{j \in J} \lambda_{j} f_{j}\left(x^{*}\right)
$$

for every $y \in K$. Moreover, since $\lambda_{j}+1 / n>0$ for every $n \in \mathbb{N}$ and $j \in J$ one can find by our assumption for each $n \in \mathbb{N}$ some $y^{(n)} \in K$ satisfying

$$
\sum_{j \in J}\left(\lambda_{j}+1 / n\right) f_{j}\left(y^{(n)}\right) \leq \sum_{j \in J}\left(\lambda_{j}+1 / n\right) f_{j}\left(x^{*}\right)
$$

Due to the compactness of $K$ the sequence $\left\{y^{(n)}: n \in I N\right\}$ contains a converging subsequence with limit $y^{(\infty)} \in K$ and this yields by the continuity of $f_{j}, j \in J$, and the previous observations that

$$
\sum_{j \in J} \lambda_{j} f_{j}\left(y^{(\infty)}\right) \leq \sum_{j \in J} \lambda_{j} f_{j}\left(x^{*}\right) \leq-\delta+\sum_{j \in J} \lambda_{j} f_{j}\left(y^{(\infty)}\right)
$$

Hence, we have contained a contradiction and so the desired result is proved.

The properties validating the proof of Theorem 2.4 are the compactness of the convex set $K$ and $F(K)+\mathbb{R}_{+}^{|J|}$ is a closed and convex set. Since we assume that $F$ is lower semicontinuous (see observations after Theorem 2.1 ) it follows due to the compactness of $K$ that $F(K)+\mathbb{R}_{+}^{|J|}$ is closed (see [9]) and so the key properties are the compactness of the convex set $K$ and $F(K)+\mathbb{R}_{+}^{|J|}$ is a convex set. Observe the last property is known in the literature as convex-like, $[14,15]$. If we drop the assumption that the convex set $K$ is compact the result of Theorem 2.4 does not hold as shown by the following counterexample with $K$ an unbounded convex set and $F$ a convex vector function. 
EXAMPLE 2.2 Let $X=[0, \infty) \times[0, \infty), J=\{1,2\}$ and $f_{1}\left(x_{1}, x_{2}\right)=x_{1}$ and $f_{2}\left(x_{1}, x_{2}\right)=-\sqrt{x_{1}}$.

If $K=\left\{\left(x_{1}, x_{2}\right): x_{1}>0, x_{2} \geq 1 / x_{1}\right\} \subset X$ then it is easy to show that $K$ contains an optimal solution of the problem

$$
\min \left\{\lambda_{1} x_{1}-\lambda_{2} \sqrt{x_{1}}:\left(x_{1}, x_{2}\right) \in X\right\}
$$

for any $\lambda_{1}, \lambda_{2}>0$. However, since $D_{J}((0,0))=\{(0, x): x \geq 0\}$ it follows that $D_{J}((0,0)) \cap K=\emptyset$ and so $K$ is not a $J$-dominating set.

Although the assumption that $F(K)+\mathbb{R}_{+}^{|J|}$ is a convex set is much weaker than $F$ is a convex vector valued function, it does not cover the important class of a lower semicontinuous quasiconvex vector-valued function $F$ on the convex set $K$. This is shown by the following counterexample with $K$ a compact and convex set and $F$ lower semicontinuous and quasiconvex.

Example 2.3 Let $X=\mathbb{R}^{2}, J=\{1,2\}$ and $f_{1}, f_{2}: \mathbb{R}^{2} \longrightarrow \mathbb{R}$ the lower semicontinuous quasiconvex functions

$$
f_{1}\left(x_{1}, x_{2}\right)=\left\{\begin{array}{ll}
0, & \text { if }\left|x_{2}\right| \leq-x_{1} \\
3, & \text { if } x_{1}>x_{2} \\
2, & \text { otherwise }
\end{array} \quad f_{2}\left(x_{1}, x_{2}\right)= \begin{cases}3, & \text { if } x_{1}<1 / 2 \\
0, & \text { if }\left|x_{2}\right| \leq x_{1}-1 \\
2, & \text { otherwise }\end{cases}\right.
$$

It is easy to verify by inspection that the set $\{(0,0),(1,0)\}$ contains an optimal solution of the problem $\min \left\{\lambda_{1} f_{1}(x)+\lambda_{2} f_{2}(x): x \in X\right\}$ for any $\lambda_{1}>0, \lambda_{2}>0$. Hence the compact and convex set $K=\left\{\left(x_{1}, 0\right): 0 \leq x_{1} \leq\right.$ $1\}$ satisfies the assumptions of Theorem 2.4. However, the set $D_{J}((1,2))$ is given by

$$
\left\{\left(x_{1}, x_{2}\right): x_{1} \geq 1 / 2, x_{1} \leq x_{2}\right\},
$$

and so $K \cap D_{J}((1,2))=\emptyset$ or equivalently $K$ does not belong to $\mathcal{K}_{J}$.

In the next two sections we will discuss some applications of the results derived in this section.

\section{A localization result in Linear Regression}

Let $\left(x_{1}, y_{1}\right),\left(x_{2}, y_{2}\right), \ldots,\left(x_{p}, y_{p}\right)$ be $p \geq 2$ points in the plane, not all contained in the same vertical line, and consider for each $i \in I=\{1,2, \ldots, p\}$ the value $\varepsilon_{i}$ given by

$$
\varepsilon_{i}(a, b)=\left|y_{i}-a x_{i}-b\right|
$$

In other words, $\varepsilon_{i}(a, b)$ gives the vertical distance from the point $\left(x_{i}, y_{i}\right)$ to the line $y=a x+b$. 
Most regression estimates are obtained by solving mathematical programs of the form

$$
\min _{(a, b) \in \mathbb{R}^{2}} \Phi\left(\varepsilon_{1}(a, b), \varepsilon_{2}(a, b), \ldots, \varepsilon_{p}(a, b)\right)
$$

for some nondecreasing function $\Phi$ that aggregates the error at the different points.

It has been shown in [16] that the set $E_{I}$ of Pareto-optimal solutions of the vector-optimization problem

$$
\text { vector- } \min _{(a, b)}\left(\varepsilon_{1}(a, b), \varepsilon_{2}(a, b), \ldots, \varepsilon_{p}(a, b)\right)
$$

is an $I$-dominating set. However, the set $E_{I}$ is in general not convex (it is just a connected union of polytopes) which makes this set of limited interest for optimization purposes. Our next result shows that a more manageable $I$-dominating set can be built with the techniques presented in the previous section.

Lemma 3.1 For each $i, j \in I$ such that $x_{i} \neq x_{j}$, let $y=a_{i j} x+b_{i j}$ be the line passing through $\left(x_{i}, y_{i}\right)$ and $\left(x_{j}, y_{j}\right)$. If $K=\operatorname{conv}\left(\left\{\left(a_{i j}, b_{i j}\right): 1 \leq i<j \leq\right.\right.$ $\left.\left.p, x_{i} \neq x_{j}\right\}\right)$ then the compact and convex set $K$ belongs to $\mathcal{K}_{I}$. Furthermore, for any convex set $K^{*} \in \mathcal{K}_{I}$ it follows that $K \subset K^{*}$.

Proof. Observe first, for any $i, j \in I, i \neq j$ and $t \in[0,1]$, that the set $K$ contains an optimal solution to the problem $\min _{(a, b)} t \varepsilon_{i}(a, b)+(1-t) \varepsilon_{j}(a, b)$ (in fact, the set of vertices of $K$ enjoys this optimality property). Hence, as $K$ is compact, and each $\varepsilon_{i}$ is convex, Theorem 2.4 implies that $K$ is a $J$-dominating set for all $J \subset I,|J|=2$, and hence by Theorem 2.3 the set $K$ is $I$-dominating.

On the other hand, if $K^{*}$ is a convex $I$-dominating set, it must contain the points $\left(a_{i j}, b_{i j}\right)$ which are the unique points that make both $\varepsilon_{i}$ and $\varepsilon_{j}$ simultaneously 0 . Hence, by the definition of $K$ it follows that $K \subset K^{*}$.

REMARK 3.1 The geometrical description of the set $E_{I}$ given in [16] enables to show that the set $K$ defined in Lemma 3.1 is the convex hull of $E_{I}$. The above lemma also shows that $K$ is minimal among all convex $I$-dominating sets.

\section{A localization result in Location}

In planar single-facility location models, a family of users (usually represented as points in $\mathbb{R}^{2}$ ) is given, and one seeks the location $x \in \mathbb{R}^{2}$ for 
a facility such that the transportation costs from $x$ to the users are minimized, [17]. Transportation costs from the facility to each user are assumed to be increasing in the distance, leading typically to nonconvex nonsmooth optimization problems, [18].

The statement of localization results for these problems has attracted the attention of many researchers (see e.g. [6, 19, 20, 21, 7] mainly due to two reasons: First, a dominating set provides a broad-sense sensitivity analysis, and also, the most popular resolution method, the $\operatorname{BSSS}([22,23])$, is a Branch and Bound procedure, which requires as a first step the construction of a compact set containing an optimal solution.

The most relevant localization theorem states (see Corollary 1 of [7]) that $\operatorname{clconv}(A)$ is a dominating set for the family of functions $\{\gamma(x-a)\}_{a \in A}$ with $\gamma$ a norm on $\mathbb{R}^{2}$ and $A \subseteq \mathbb{R}^{2}$. To extend and reobtain this result we denote by $\gamma_{S}(x)$ the distance from $x$ to the closest point in a nonempty, compact and convex set $S$, i.e.

$$
\gamma_{S}(x):=\min _{s \in S} \gamma(x-s)
$$

We will now present a localization result for the functions $\left\{\gamma_{A_{i}}(x)\right\}_{i \in I}$ with $A_{i}, i \in I$, a collection of nonempty, compact and convex sets. To prove this result, we first present a lemma which extends the well-known majority theorem of Witzgall ([24]).

Lemma 4.1 Let $A_{1}, A_{2}, \ldots, A_{p}$ be nonempty, compact and convex subsets of $\mathbb{R}^{2}$, and consider for each $\lambda \in \mathbb{R}_{+}^{p}, \lambda \neq 0$, the problem

$$
\min _{x \in \mathbb{R}^{2}} F_{\lambda}(x)=\sum_{i=1}^{p} \lambda_{j} \gamma_{A_{j}}(x)
$$

If there exists some $1 \leq k \leq p$ such that $\lambda_{k} \geq \sum_{i \neq k} \lambda_{i}$, then an optimal solution to $\left(L_{\lambda}\right)$ belongs to $A_{k}$.

Proof. Let $x \in \mathbb{R}^{2}$ and $a_{i} \in A_{i}, i \in I$, be such that $\gamma_{A_{i}}(x)=\gamma\left(x-a_{i}\right)$. By the properties of a norm it follows that

$$
\begin{array}{rlc}
F_{\lambda}(x) & = & \sum_{i \neq k} \lambda_{i} \gamma_{A_{i}}(x)+\lambda_{k} \gamma_{A_{k}}(x) \\
& \geq & \sum_{i \neq k} \lambda_{i}\left(\gamma_{A_{i}}(x)+\gamma_{A_{k}}(x)\right) \\
& = & \sum_{i \neq k} \lambda_{i}\left(\gamma\left(x-a_{i}\right)+\gamma\left(x-a_{k}\right)\right) \\
\geq & \sum_{i \neq k} \lambda_{i} \gamma\left(a_{k}-a_{i}\right) \\
\geq & \sum_{i \neq k} \lambda_{i} \gamma_{A_{i}}\left(a_{k}\right) \\
& = & F_{\lambda}\left(a_{k}\right) \geq \min _{a \in A_{k}} F_{\lambda}(a),
\end{array}
$$

and this proves the desired result.

Using the above lemma one can now show the following result. 
LEMMA 4.2 Let $I$ be an arbitrary nonempty index set and $A_{i}, i \in I$, a collection of nonempty, compact and convex sets. If for each $i, j \in I, i \neq j$, the vector $z_{i j}$ is an optimal solution of $\min _{x \in A_{i}} \gamma_{A_{j}}(x)$ then the set $\operatorname{clconv}\left(\left\{z_{i j}\right.\right.$ : $i, j \in I, i \neq j\})$ is an I-dominating set for the family of functions $\left(\gamma_{A_{i}}(x)\right)_{i \in I}$

Proof. By Lemma 4.1 and Theorem 2.4 it follows that conv $\left\{z_{i j}, z_{j i}\right\}$ is an $\{i, j\}$-dominating set. Applying now Theorem 2.2 we obtain that $K=$ $\operatorname{clconv}\left(\left\{z_{i j}: i, j \in I, i \neq j\right\}\right)$ is an $I$-dominating set.

REMARK 4.1 If each $A_{i}, i \in I$, reduces to the single point set $\left\{a_{i}\right\}$ it follows that $z_{i j}=a_{i}$ for every $i, j \in I$. Hence, by the above lemma this yields that $\operatorname{clconv}\left(\left\{a_{i}: i \in I\right\}\right)$ is an $I$-dominating set for the family of functions $\left\{\gamma\left(x-a_{i}\right)\right\}_{i \in I}$.

\section{Conclusions}

In this note it has been shown using Helly's theorem that the problem of obtaining a dominating set for a (possibly infinite) family of convex functions can be reduced to constructing dominating sets for families with lower cardinality. This seems to be of practical interest when the number of functions involved is much higher than the dimension of the space, as is typical in Regression Estimation or Location Theory.

As an illustration of the usefulness of these techniques, convex dominating sets for linear regression and location problems have been presented. It should be noted that the same strategy can also be succesfully applied to rediscover and generalize other localization results for planar location problems. For instance, using Theorem 4.3 in [25], the minimal convex dominating set for problems with mixed polyhedral gauges is easily shown to be the convex hull of the intersection points which are strictly efficient (see [25]). On the other hand, this result, in conjunction with Theorem 6 in [26], enables us to rediscover and extend the octagonal-hull property of [20]. This shows another example of a problem in location theory to which one can apply the results of Section 2. Finally, we like to observe that the construction of dominating sets for problems from other fields is now under study.

Acknowledgements. The research of the first author has been partially financed by the Dirección General de Investigación Científica y Técnica, Spain, under grant PB93-0927. This support is gratefully acknowledged. 


\section{References}

[1] Plastria, F., The minimization of lower subdifferentiable functions under nonlinear constraints, Journal of Optimization Theory and Applications, Vol. 57, pp. 463-484, 1988.

[2] Frenk, J.B.G., Gromicho, J., And Zhang, S., A deep cut ellipsoid algorithm for convex programming: Theory and applications, Mathematical Programming, Vol. 63, pp. 83-108, 1994.

[3] Frenk, J.B.G., And Gromicho, J., An Elementary Rate of Convergence Proof for the Deep Cut Ellipsoid Method, in Recents Advances in Nonsmooth Optimization, D.Z. Du, L. Qi and R.S. Womersley (eds.), World Scientific Publishing Co. 106-120, 1995.

[4] Gromicho, J.,Quasiconvex Optimization and Location Theory, Ph.D. Thesis, Tinberger Institute Research Series 90, Thesis Publishers, Amsterdam, 1995.

[5] Horst, R., And Tuy, H., Global Optimization. Deterministic Approaches, Springer-Verlag, 1993.

[6] Plastria, F., Localization in single facility location, European Journal of Operational Research, Vol. 18, pp. 215-219, 1984.

[7] Wendell, R.E., And Hurter, A.P., Location theory, dominance, and convexity, Operations Research, Vol. 21, pp. 314-320, 1973.

[8] KuHN, H.W., On a pair of dual nonlinear programs, in J. Abadie (ed.), Methods of Nonlinear Programming, North-Holland, 37-54, 1967.

[9] Aubin, J.P., Optima and Equilibria (An Introduction to Nonlinear Analysis, Springer-Verlag, Berlin, 1993.

[10] Baggett, L.W., Functional Analysis, Marcel-Dekker, New York, 1992.

[11] Rockafellar, R.T., Convex Analysis, Princeton University Press, Princeton, 1972.

[12] Hiriart-Urruty, J.B., And Lemarechal, C. Convex Analysis and Minimization Algoritms, volume I, Springer-Verlag, Berlin, 1993.

[13] Dem'yanov, V.F., and Vasil'Ev, L.V., Nondifferentiable Optimization, Optimization Software inc, 1985. 
[14] Hayashi, M., and Komiya, H., Perfect duality for convexlike programs, Journal of Optimization Theory and Applications, Vol. 38, pp. 179-189, 1982.

[15] IllÉS, T., AND KASSAY, G., Farkas type theorems for generalized convexities. P.U.M.A., Vol. 5, pp. 225-239, 1994.

[16] Carrizosa, E., Conde, E., Fernández, F.R., MuñozMárquez, M., and Puerto, J., Pareto-optimality in Linear Regression. Journal of Mathematical Analysis and Applications, Vol. 190, pp. 129-141, 1995.

[17] Plastria, F., Continuous location problems, in Facility location (Z. Drezner, ed.), Springer-Verlag, New York, 1995.

[18] Hansen, P., Jaumard, B., And Tuy, H., Global optimization in location, in Facility location (Z. Drezner, ed.), Springer-Verlag, New York, 1995.

[19] Durier, R., And Michelot, C., Set of efficient points in a normed space, Journal of Mathematical Analysis and Applications, Vol. 117, pp. 506-528, 1986.

[20] Hansen, P., Perreur, J, and Thisse, J.F., Location theory, dominance, and convexity: Some further results, Operations Research, Vol. 28, pp. 1241-1250, 1980.

[21] Juel, H., AND Love, R.F., Hull properties in location problems, European Journal of Operational Research, Vol. 12, pp. 262-265, 1983.

[22] Hansen, P., Peeters, D., Richard, D., and Thisse, J.F., The minisum and minimax location problems revisited, Operations Research, Vol. 33, pp. 1251-1265, 1985.

[23] Plastria, F., GBSSS: The Generalized Big Square Small Square method for planar single-facility location, European Journal of Operational Research, Vol. 62, pp. 163-174, 1992.

[24] Witzgall, C.J., Optimal location of a central facility: mathematical models and concepts, National Bureau of Standards report 8388, US Department of Commerce, Washington D.C., USA, 1964.

[25] Durier, R., And Michelot, C., Geometrical properties of the Fermat-Weber problem, European Journal of Operational Research, Vol. 20, pp. 332-343, 1985. 
[26] Carrizosa, E., and Fernández, F.R., El conjunto eficiente en problemas de localización con normas mixtas $\left(L_{p}\right)$, Trabajos de Investigación Operativa, Vol. 6, pp. 61-69, 1991.

\section{E. CARRIZOSA}

Facultad de Matemáticas. Universidad de Sevilla.

Tarfia s/n. 41012 Sevilla, Spain.

\section{J.B.G. FRENK}

Econometric Institute, Erasmus Universiteit Rotterdam.

P.O. Box 1738, NL - 3000. DR Rotterdam, The Netherlands. 